\section{Abstractions}

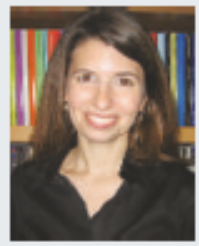

FIRST AUTHOR
On page 412, Leslie
Osborne and her
colleagues report the
results of experiments to
investigate whether
variability in fine
movements is the result of sensory or motor errors. The group trained monkeys to visually track spots of light moving on a monitor, and measured their eye movements. Then they an alysed the variability in the movement and found that it could be explained by errors in the sensory estimate of the target, as opposed to noise in the motor system itself.

Osborne, aneurobiology postdoc at the University of California, San Francisco, has a background in biophysics and a postdoc in neuroscience. This range of skills helped her to serve as a conduit between Steve Lisber ger, her biologist principal investigator, and Bill Bialek, her theoretical physics mentor. Nature found outmore about her bridging role.

How did you cross from physics to biology? At Berkeley, where I did my PhD, I spent a lot of my time in alaser-physics lab building a device to measuresmallmovements. I used this to study how tiny hairs on crickets help the insects to detect predators.

What were theroles of the other authors? The work grew out of conversations I had with Bill about variability in trackingeye movements and whether we could look for evidence that any variability seen could be explained by noise in thesensory system. I did the experiments. Bill provided the theoretical framework and I analysed all the data. Steve is great at making you define your idea, until you think that your conclusions are not just a mathematical construct He was our resident sceptic.

What was it like to workcross-discipline? Thebiggest challenge was that Bill and Steve speak verydifferent languages. Writing the paper was tricky - that's when you seehow different thelanguages of the two disciplines can be. Bill is comfortable expressing himself in mathematics. Biologists and neuroscientists don't tend to communicate this way. Wetookmost of the maths out of our paper - there were five pages of maths that didn'tmake it in to the final submission.

Why does biology need more theory? Because it's theory that drives new experiments. If you have an overview on how asystem might work, it allows you to ask more shrewd questions that might proveor disprove your hypothesis.

What's next?

To see if our finding generalizes toothermotor systems. Evertually, I would like to track neural activity along with behaviour to watch the brain's translation of vision to actionemerge.
MAKING THE PAPER

Shehzaad Kaka

and Fred Mancoff

Power from some devices can be

more than the sum of their parts.

Fireflies flashing in unison...pedestrians marching over bridges and falling in step with each other... Nature has a way of getting things in synch. So it should come as no surprise that two separate groups - one academic, one industrial - simultaneously pursued such effects in tiny devices (see pages 389 and 393).

The starting point for both groups was an earlier finding that nanosized magnetic oscillators can generate microwaves. By combining multiple devices, the teams hoped to generate sizeable amounts of microwave power. To test out this idea, they set about linking two devices. This, they hope, will serve as the first step towards building a simple wireless circuit, which could pave the way to faster computation.

Selecting and assembling the building blocks for their devices posed difficult problems for both groups. The fabrication process was "fairly complicated", says Shehzaad Kaka, first author on the paper from the public effort, based at the US National Institute of Standards and Technology (NIST) laboratory in Boulder, Colorado. Fred Mancoff, first author with the commercial group at Freescale Semiconductor in Chandler, Arizona, agrees.

The devices are made up of several layers of thin-film materials, and patterning the tiny magnetic oscillators meant using electron beam lithography to resolve the features between neighbouring oscillators. They had to find ways to monitor how the devices worked, both as individuals and when the two were hooked up together. "At first, we weren't really set up for measuring these high-frequency signals, ${ }^{,}$Mancoff says.

As the two groups moved from studying individual oscillators to two coupled devices, they took slightly different approaches. For each set of measurements, the NIST group

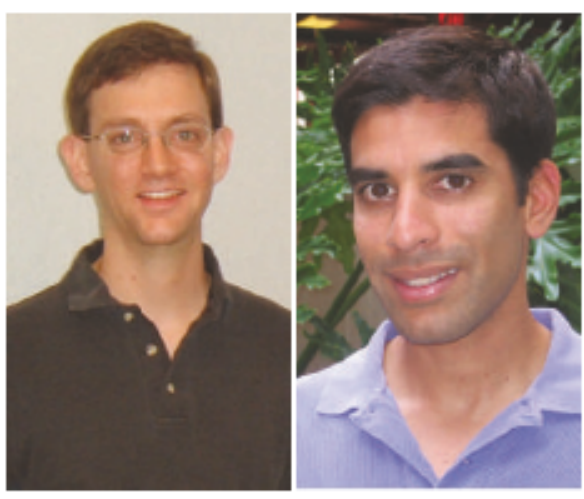

Fred Mancoff (left) and Shehzaad Kaka.

kept its two oscillators in the same position. The Freescale researchers focused instead on what happened as they changed the distance between the two devices.

Despite the different approaches, both groups quickly saw the same thing: at some point, the electrical output signal from each of the two devices began oscillating at the same frequency. "It was something we were hoping to get," says Kaka.

Both teams were surprised at how quickly they got a result. "From the first step, there was a fairly strong effect going on as a result of the spacing," Mancoff says. "I knew that there was probably some interesting physics going on."

Each of the two groups was aware that the other was doing similar research - not surprising as the two first authors had shared an office at NIST when Kaka was a PhD student and Mancoff was a postdoc. So, having validated their work with more experiments, they both scrambled to submit a paper. ${ }^{\alpha}$ Once we found that Freescale was also intending to publish, that definitely motivated us to finish up, write it up and send it in for publication," says Kaka.

Although neither group demonstrated that the devices in tandem can generate sizeable amounts of power, both groups aim to combine more devices. Thanks to the potential applications of the devices, the groups expect to see even more competition in this field. And they might even see more developments that seem to happen in synch.

\title{
QUANTIFIED CHILE
}

\section{A numerical pers pective on Nature authors.}

In Chile, pursuing science at a regional university such as the Pontifical Catholic University of Valparaiso is noteasy, says Marco Cisternas. The size and influence of competing universities in Santiago, the country'scapital, means that funding from government agencies is hard to come by for scientists in smaller cities.

Cistemas is lucky to have had full support for his research, but really, he says, the anly way to work is to team up withother national universities and with foreign colleagues. Cisternas is a mermber of a multidisciplinary team with scientists from the United States, Japan, India and Indonesia, as well as Chile. The group works on earthquakes, and its most recent research maps out giant-earthquake recurrence over the past two millennia in southem-central Chile (seepage 404).
10 authors of paperspublished in Nature so far this year are working in Chile (total number of published authors $=3,984$ ).

3 Naturepapers published this year have contributing authors working in Chile (total number ofpublished papers $=599$ ).

14 Submissions to Nature this year have come from Chile.

3 authors from the Pontifical Catholic University of Valparaiso have research published in Nature this week. 